Random Oper. and Stoch. Equ., Vol. 13, No. 1, pp. 11-16 (2005)

(c) VSP 2005

\title{
On Selberg's beta integrals
}

A. K. GUPTA ${ }^{1}$, D. G. $\mathrm{KABE}^{2}$

${ }^{1}$ Department of Mathematics and Statistics

Bowling Green State University, Bowling Green, Ohio 43403-0221, USA

${ }^{2}$ St. Mary's University

Halifax , NS , CANADA B3H 3C3

Received for ROSE February 16, 2004

Abstract - Askey and Richards (1989) evaluate Selberg's first and second beta integrals using Aomoto's (1987) formidable methodology of setting and solving a first order difference equation. Using this methodology they evaluate certain other beta and gamma type integrals. However, Selberg's first and second beta and gamma type integrals very elegantly fit within the framework of hypercomplex multivariate normal distribution theory developed by Kabe (1984), and hence can be evaluated using the known multivariate normal distribution theory integrals.

Key Words and Phrases: Selberg's first beta integral; hypercomplex normal multivariate distribution theory; Wishart density; multivariate beta density; roots density; Stirling's integral.

\section{INTRODUCTION}

Let $\Lambda$ be a $p \times p$ diagonal matrix, then Selberg's first beta integral states that

$$
\int|\Lambda|^{g-1}|I-\Lambda|^{h-1} \prod_{i<j}\left(\lambda_{i}-\lambda_{j}\right)^{2 k} d \Lambda=\prod_{i=1}^{p} \frac{\Gamma(g+(i-1) k) \Gamma(h+(i-1) k) \Gamma(1+i k)}{\Gamma(g+h+(p+i-2) k) \Gamma(1+k)} .
$$

Selberg's second beta integral states that

$$
\begin{aligned}
\int|\Lambda|^{g-1}(1-t r \Lambda)^{h-1} \prod_{i<j} & \left(\lambda_{i}-\lambda_{j}\right)^{2 k} d \Lambda \\
& =\frac{\Gamma(h) \pi^{\frac{1}{2} k p(p-1)}}{\Gamma(h+p g+(p-1) p k)} \prod_{i=1}^{p} \frac{\Gamma(g+(p-i) k) \Gamma(1+i k)}{\Gamma(1+k)} .
\end{aligned}
$$

The integrals (1) and (2) fit very elegantly within the framework of hypercomplex (HC) multivariate normal distribution theory. Thus we evaluate these integrals using this theory.

The next section records some useful results, and section 3 evaluates the integral (1). In section 4 we evaluate the integral (2).

AMS 2000 subject classifications: Primary 62H10; Secondary 33A15. 


\section{SOME USEFUL RESULTS}

Kabe (1984) develops the HC normal multivariate distribution theory as follows. Let $X_{1}, X_{2}, \ldots, X_{4 t}, t=\frac{1}{4}, \frac{1}{2}, 1,2$ be $4 t(p \times n)$ real random matrices having the $4 p n t$ variate normal density

$$
(4 \pi t)^{-2 p n t}\left|\Sigma_{0}\right|^{-\frac{1}{2} n} \exp \left\{-\operatorname{tr} \Sigma_{0}^{-1} X X^{\prime} / 4 t\right\}
$$

where $X=\left(X_{1}^{\prime}, X_{2}^{\prime}, \ldots, X_{4 t}^{\prime}\right)^{\prime}$, and $\Sigma_{0}(4 t \times 4 t)$ is

$$
\left[\begin{array}{cccccccc}
\Sigma_{1} & -\Sigma_{2} & -\Sigma_{3} & -\Sigma_{4} & -\Sigma_{5} & -\Sigma_{6} & -\Sigma_{7} & -\Sigma_{8} \\
\Sigma_{2} & \Sigma_{1} & -\Sigma_{4} & \Sigma_{3} & -\Sigma_{6} & \Sigma_{5} & \Sigma_{8} & -\Sigma_{7} \\
\Sigma_{3} & \Sigma_{4} & \Sigma_{1} & -\Sigma_{2} & -\Sigma_{7} & \Sigma_{8} & \Sigma_{5} & \Sigma_{6} \\
\Sigma_{4} & -\Sigma_{3} & \Sigma_{2} & \Sigma_{8} & -\Sigma_{8} & \Sigma_{7} & -\Sigma_{6} & \Sigma_{3} \\
\Sigma_{5} & \Sigma_{6} & \Sigma_{7} & \Sigma_{8} & \Sigma_{1} & -\Sigma_{2} & -\Sigma_{3} & -\Sigma_{4} \\
\Sigma_{6} & -\Sigma_{5} & \Sigma_{8} & -\Sigma_{7} & \Sigma_{2} & \Sigma_{1} & \Sigma_{4} & \Sigma_{3} \\
\Sigma_{7} & -\Sigma_{8} & -\Sigma_{5} & \Sigma_{6} & \Sigma_{3} & -\Sigma_{4} & \Sigma_{1} & \Sigma_{2} \\
-\Sigma_{8} & \Sigma_{7} & -\Sigma_{6} & -\Sigma_{5} & \Sigma_{4} & \Sigma_{3} & -\Sigma_{2} & \Sigma_{1}
\end{array}\right]
$$

where now $t=2$, and we use Hamilton's octonioins, Halberstam and Ingram (1967, p. 654 , equatin (1)).

In the octonion case $\Sigma_{1}$ is a $p \times p$ real positive definite symmetric matrix; and $\Sigma_{2}, \ldots, \Sigma_{8}$ are real $p \times p$ skew symmetric matrices. Now we set

$$
Y=X_{1}+i X_{2}+j X_{3}+k X_{4}+l X_{5}+m X_{6}+n X_{7}+r X_{8}
$$

where the octonions $i, j, k, l, m, n, r$ satisfy the multiplication rule $i^{2}=j^{2}=k^{2}=l^{2}=m^{2}=n^{2}=r^{2}=-1=i j k=i l n=i r n=j l n=j m r=k i r=k n m$,

and we observe that

$$
\bar{Y}=X_{1}-i X_{2}-j X_{3}-k X_{4}-l X_{5}-m X_{6}-n X_{7}-r X_{8}
$$

is the octonion conjugate of $Y$, and

$$
d Y=d X_{1} d X_{2} \ldots d X_{8}
$$

After some formidable matrix algebra, Kabe (1984) writes the $p n$ variate HC multivariate normal density of $Y$ to be

$$
g(Y)=\pi^{-2 p n t}|\Sigma|^{-2 n t} \exp \left\{-\operatorname{tr} \Sigma^{-1} Y \bar{Y}^{\prime}\right\}
$$

where

$$
\Sigma=\Sigma_{1}+i \Sigma_{2}+j \Sigma_{3}+k \Sigma_{4}+l \Sigma_{5}+m \Sigma_{6}+n \Sigma_{7}+r \Sigma_{8} .
$$

Next setting $Y \bar{Y}^{\prime}=S$, the HC Hermitian $p \times p$ matrix, Kabe (1984, p. 67, equation (14)) shows the HC Wishart density of $S$ to be

$$
g(S)=\left\{\Gamma_{p}(2 n t)\right\}^{-1}|\Sigma|^{-2 n t}|S|^{2 t(n-p+1)-1} \exp \left\{-t r \Sigma^{-1} S\right\},
$$

where (Gupta and Nagar, 2000; p. 19)

$$
\Gamma_{p}(a)=\pi^{t p(p-1)} \prod_{i=1}^{p} \Gamma(a-2 t(p-i)) .
$$


If $S$ and $R$ are two $p \times p$ HC Hermitian matrix and $H$ is a $p \times p$ HC matrix, then the Jacobian of the transformation of the relation $S=H R \bar{H}^{\prime}$ is

$$
J(S: R)=\left|H \bar{H}^{\prime}\right|^{2 t(p-1)+1} .
$$

Finally, if the joint density of two $p \times p$ HC Hermitian matrices is given by

$$
g(A, B)=K \exp \{-\operatorname{tr}(A+B)\}|B|^{2 t(n-p+1)-1}|A|^{2 t(q-p+1)-1},
$$

where $K$ denotes the corresponding constant, then the density of the $p \times p$ HC Hermitian matrix $R$ defined by relation

$$
R=G^{-\frac{1}{2}} A G^{-\frac{1}{2}}, \quad A+B=G
$$

is easily found by using (10) to be

$$
g(R)=\left\{B_{p}(2 n t, 2 q t)\right\}^{-1}|I-R|^{2 t(n-p+1)-1}|R|^{2 t(q-p+1)-1},
$$

where (Gupta and Nagar, 2000, p. 20)

$$
B_{p}(a, b)=\Gamma_{p}(a) \Gamma_{p}(b) / \Gamma_{p}(a+b) .
$$

If now $\Lambda$ is the diagonal matrix of the roots of $R$ of $(13)$, then Kabe $(1984$, p. 68 , equation (21)) shows the Jacobian of the transformation from $R$ to $\Lambda$, for all distribution theory purposes, is

$$
J(R: \Lambda)=\prod_{i<j=1}^{p}\left(\lambda_{i}-\lambda_{j}\right)^{4 t}
$$

Thus the density of $\Lambda$ is

$$
g(\Lambda)=\left\{B_{p}(2 n t, 2 q t)\right\}^{-1}|I-\Lambda|^{2 t(n-p+1)-1}|\Lambda|^{2 t(q-p+1)-1} \prod_{i<j=1}^{p}\left(\lambda_{i}-\lambda_{j}\right)^{4 t} .
$$

Now setting $2 t=k$, we note that (16) is the same, although not exactly the same, integral as (1). Thus the integral (1) can be evaluated by using (16).

\section{SELBERG'S FIRST BETA INTEGRAL}

We note that

$$
\begin{aligned}
& \Gamma_{p}(n k)=\pi^{\frac{1}{2} k p(p-1)} \prod_{i=1}^{p} \Gamma(n k+k i-k p), \\
& \Gamma_{p}(k q)=\pi^{\frac{1}{2} k p(p-1)} \prod_{i=1}^{p} \Gamma(k q+k i-k p),
\end{aligned}
$$

and setting

$$
k q=g+k p-k, \quad n k=h+k p-k,
$$


we have the result from (16) that

$$
\int|I-\Lambda|^{h-1}|\Lambda|^{g-1} \prod_{i<j=1}^{p}\left(\lambda_{i}-\lambda_{j}\right)^{2 k} d \Lambda=\frac{\left.\prod_{i=1}^{p} \Gamma\left(h_{(} i-1\right) k\right) \prod_{i=1}^{p} \Gamma(g+(i-1) k)}{\prod_{i=1}^{p} \Gamma(h+g+(p+i-2) k)} .
$$

Note that (20) is not the result (1). To derive (1) from (20), we note from (8) that

$$
\int \exp \{-\operatorname{tr} \Lambda\}|\Lambda|^{g-1} \prod_{i<j=1}^{p}\left(\lambda_{i}-\lambda_{j}\right)^{2 k} d \Lambda=\prod_{i=1}^{p} \Gamma(g+(i-1) k) .
$$

In the integral (2) and (21) the roots are ordered, but the variables of integration in (1) are unordered. Thus, e.g., the unordered variable integral (21) must be written as

$$
\int \exp \{-\operatorname{tr} \Lambda\}|\Lambda|^{g-1} \prod_{i<j=1}^{p}\left(\lambda_{i}-\lambda_{j}\right)^{2 k} d \Lambda=\prod_{i=1}^{p} \Gamma(g+(i-1) k) f(i k) .
$$

However Stirling's integral states that

$$
\int \exp \{-\operatorname{tr} \Lambda\}|\Lambda|^{g-1} \prod_{i<j=1}^{p}\left(\lambda_{i}-\lambda_{j}\right)^{2 k} d \Lambda=\prod_{i=1}^{p} \frac{\Gamma(g+(i-1) k) \Gamma(1+i k)}{\Gamma(1+k)}
$$

Obviously (1) follows from (20) and (23), because $f(i k)$ is the same for (20) and (21).

\section{SELBERG'S SECOND BETA INTEGRAL}

For a $p \times p$ diagonal matrix $\Lambda, 0<\Lambda<I$, Selberg's second beta integral is defined by the identity

$$
\begin{aligned}
\int|\Lambda|^{g-1}(1-\operatorname{tr} \Lambda)^{h-1} \prod_{i<j}^{p}\left(\lambda_{i}-\lambda_{j}\right)^{2 k} d \Lambda \\
=\frac{\pi^{\frac{1}{2} k p(p-1)} \Gamma(h)}{\Gamma((g+k p-k) p+h)} \prod_{i=1}^{p} \frac{\Gamma(g+(i-1) k) \Gamma(1+i k)}{\Gamma(1+k)} .
\end{aligned}
$$

Askey and Richards (1989) establish (24) by using Aomoto's (1987) formidable methodology of solving a certain first order difference equation. We simply and elegantly evaluate (24) using HC multivariate normal distribution theory results due to Kabe (1984).

If $S$ is a $p \times p \mathrm{HC}$ Hermitian matrix having the $\mathrm{HC}$ octonion Wishart density

$$
g(S)=[\Gamma(2 p n t)]^{-1}|S|^{2 t(n-p+1)-1} \exp \{-\operatorname{tr} S\}
$$

then evaluating (24) reduces to the evaluation of the integral

$$
\int|S|^{2 t(n-p+1)-1}(1-\operatorname{tr} S)^{h-1} d S
$$


To evaluate (26) we use the following integral repeatedly. If $x$ is an $n$ component $\mathrm{HC}$ column vector, and $A$ a $p \times p$ HC Hermitian matrix, then Kabe (1984, p. 67, equation (15)) shows that

$$
\int_{\bar{x}^{\prime} A x=u} f\left(\bar{x}^{\prime} A x\right) d x=\frac{\pi^{2 t n}}{\Gamma(2 t n)}|A|^{-2 t} u^{2 n t-1} .
$$

Next setting

$$
S=\left[\begin{array}{cc}
s_{11} & S_{1}^{\prime} \\
S_{1} & S_{22}
\end{array}\right], \quad S_{22}(p-1) \times(p-1)
$$

and writing (26) as

$$
\int\left(s_{11}-S_{1}^{\prime} S_{22}^{-1} S_{1}\right)^{2 t(n-p+1)-1}(1-\operatorname{tr} S)^{n-1} d s_{11} d S_{1} d S_{22}
$$

and using (27), we reduce (29) to

$$
\begin{aligned}
\frac{\pi^{2 t(p-1)}}{\Gamma(2 t(p-1))} \int & \left(s_{11}-u\right)^{2 t(n-p+1)-1} u^{2 t(p-1)-1}\left|S_{22}\right|^{2 t(n-p+2)-1} \\
& \times(1-\operatorname{tr} S)^{h-1} d s_{11} d u d S_{22} \\
= & \frac{\pi^{2 t(p-1)} \Gamma(2 t(n-p+1))}{\Gamma(2 n t)} \int s_{11}^{2 n t-1}\left|S_{22}\right|^{2 t(n-p+2)-1} \\
& \times(1-t r S)^{h-1} d s_{11} d S_{22} \\
= & \frac{\pi^{t p(p-1)}}{[\Gamma(2 n t)]^{p}} \prod_{i=1}^{p} \Gamma(2 t(n-p+i)) \int\left(s_{11} s_{22} \ldots s_{p p}\right)^{2 n t-1} \\
& \times\left(1-\left(s_{11}+\ldots+s_{p p}\right)\right)^{h-1} d s_{11} \ldots d s_{p p} \\
= & \frac{\pi^{t p(p-1)} \Gamma(h)}{\Gamma(h+2 p n t)} \prod_{i=1}^{p} \Gamma(2 t(n-p+i)) .
\end{aligned}
$$

If $p \times p$ diagonal matrix $\Lambda$ is the matrix of roots of $S$, then (26) reduces to the integral

$$
\begin{aligned}
\int|\Lambda|^{2 t(n-p+1)-1}(1-\operatorname{tr} \Lambda)^{h-1} & \prod_{i<j=1}^{p}\left(\lambda_{i}-\lambda_{j}\right)^{4 t} d \Lambda \\
& =\frac{\pi^{t p(p-1)}}{\Gamma(h+2 p n t)} \prod_{i=1}^{p} \Gamma(2 t(n-p+i)) .
\end{aligned}
$$

Setting $k=2 t, n k=g+k p-k$, we write $(31)$ as

$$
\begin{aligned}
\int|\Lambda|^{g-1}(1-t r \Lambda)^{h-1} & \prod_{i<j=1}^{p}\left(\lambda_{i}-\lambda_{j}\right)^{2 k} d \Lambda \\
= & \frac{\pi^{\frac{1}{2} k p(p-1)}}{\Gamma((g+k p-k) p+h)} \prod_{i=1}^{p} \Gamma(g+(i-1) k) .
\end{aligned}
$$

Note the roots $\lambda$ 's in (32) are odered as $0<\lambda_{p}<\ldots<\lambda_{1}<1$. If this order is discarded then by Stirling's integral (32) yields (24). 


\section{REFERENCES}

1. Aomoto, K. Jacobi polynomials associated with Selberg's integrals. SIAM J. Math. Anal. 18, 545-549 (1987).

2. Askey, Richard and Richards, Donald. Selberg's second beta integral and an integral of Mehta, in Probability, Statistics, and Mathematics. Academic Press, New York (Karlin Volume) (1989).

3. Gupta, A. K. and Nagar, D. K. Matrix Variate Distributions. Chapman \& Hall/CRC, Boca Raton (2000).

4. Halberstam, H. and Ingram, R. E. The Mathematical Papers of Sir William Hamilton, Vol. III. Cambridge University Press, London (1967).

5. Kabe, D. G. Classicial statistical analysis based on a certain hypercomplex multivariate normal distribution. Metrika 31, 63-76 (1984). 
Copyright of Random Operators \& Stochastic Equations is the property of VSP International Science Publishers and its content may not be copied or emailed to multiple sites or posted to a listserv without the copyright holder's express written permission. However, users may print, download, or email articles for individual use. 